\title{
African Eyes on the Sky: The Southern African Large Telescope
}

\author{
Petri Väisänen ${ }^{1,2}$ and David A. H. Buckley ${ }^{1,2}$ \\ ${ }^{1}$ South African Astronomical Observatory, \\ P.O. Box 9, Observatory 7935, South Africa \\ email: petri@saao.ac.za, dibnob@saao.ac.za \\ ${ }^{1}$ Southern African Large Telescope Foundation, \\ P.O. Box 9, Observatory 7935, South Africa
}

\begin{abstract}
A 10-metre class telescope project on African soil is ending its commissioning period and is transitioning to science mode. We present the current status of the Southern African Large Telescope and its instruments. We will also briefly discuss the SALT capabilities for extragalactic astronomy in particular.
\end{abstract}

Keywords. telescopes, instrumentation: spectrographs

\section{Introduction}

The 10-m Southern African Large Telescope (SALT; Buckley et al. 2006) was built at the existing Sutherland observing station of the South African Astronomical Observatory (SAAO), on a $\sim 1800 \mathrm{~m}$ plateau in the semi-desert of Karoo approximately $400 \mathrm{~km}$ NorthEast of Cape Town. The primary mirror of the telescope consists of 91 identical hexagonal segments with spherical surfaces, and directs light to a spherical aberration corrector (SAC) which delivers a $\sim 10$ ' field of view. The SAC is mounted on a moving tracker at the prime focus, where also the first two instruments are located. The telescope is a fixed-altitude system, the tracker allowing continuous 1 to 3 hour tracking depending on the declination of the target.

SALT is owned by an international consortium with South Africa as a major partner with a $35 \%$ share of the observing time, and SAAO tasked with the responsibility of operating the facility. Individual scientists from all over the African continent are encouraged to collaborate with their South African colleagues to gain access to SALT, by far the largest and most modern optical astronomical facility in the region. SALT operates purely in a queue-scheduled service mode, thus no travelling to the observing site is required. Furthermore, SALT Astronomy Operations have developed advanced datadelivery and reduction pipelines (Crawford et al. 2010). Data are delivered to the users within $24 \mathrm{~h}$ of observations, they are always pre-reduced (bias, flat, gain, etc. corrected), and in many observing modes, such as long-slit spectroscopy or high time-resolution photometry, fully reduced to a level where the user can immediately begin utilizing the data products for scientific analysis.

\section{SALT status in early 2011}

The construction phase of SALT and its first two first-light instruments was completed in November 2005, followed by a period of commissioning, performance verification and early science, which extended through to early 2009. During this phase two serious problems were uncovered, one regarding the telescope's image quality (O'Donoghue et al. 


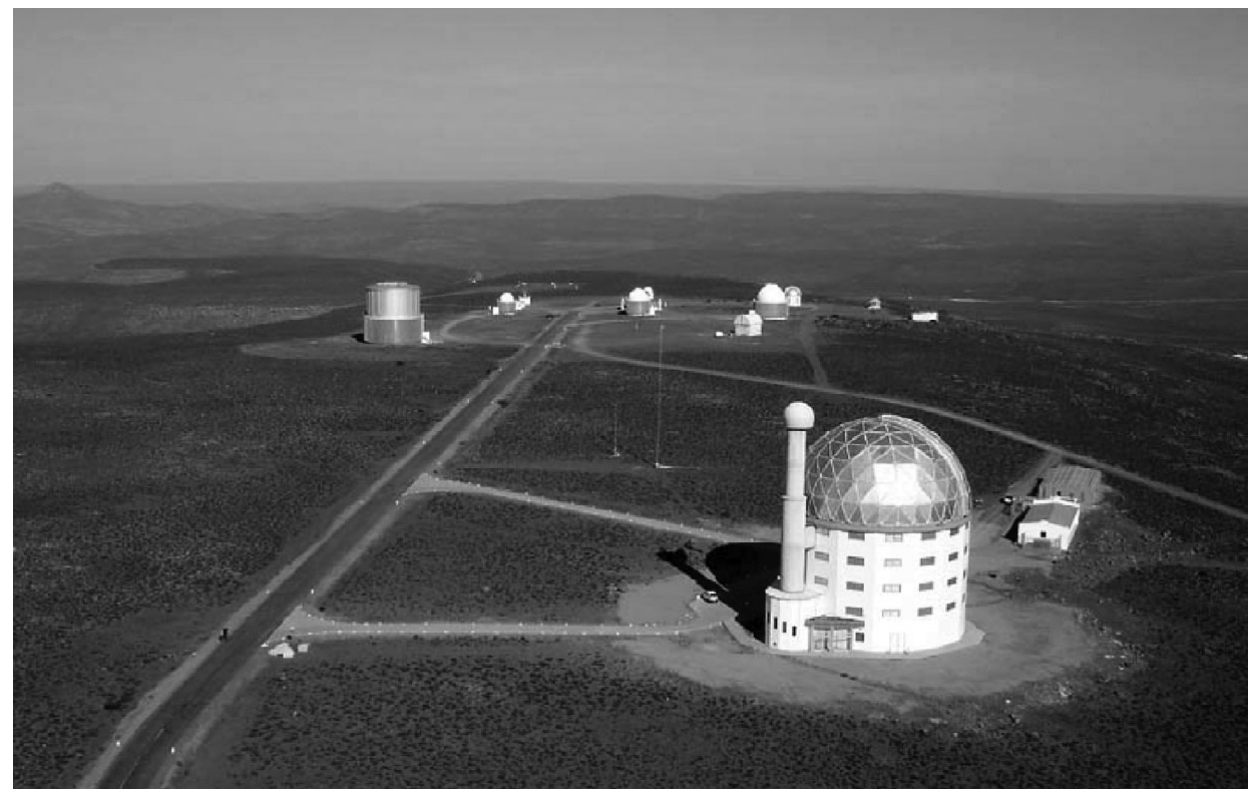

Figure 1. The 10-m SALT telescope, in the foreground, is located near the town of Sutherland, at an observatory operated by SAAO. The site is $400 \mathrm{~km}$ North-East of Cape Town in the Karoo semi-desert at an altitude of $1800 \mathrm{~m}$. The second largest telescope on the observing plateau is the 1.9-m Radcliffe telescope (left) built in the 1930's, while there is a constantly growing number of smaller modern 1-m class (robotic) telescopes built at the site.

2008) and the other related to the throughput of the major first-light instrument, the Robert Stobie Spectrograph (RSS) (Burgh et al. 2003, Nordsieck et al. 2010). The former, seen as field dependent aberrations, was subsequently traced primarily to an imperfect interface between the spherical aberration corrector and the telescope. The SAC interface had to be modified (O'Donoghue et al. 2010), and was succesfully re-installed back to the telescope in August 2010. The RSS throughput problems, which were particularly evident in the blue $(<400 \mathrm{~nm})$, were on the other hand found to be a result of subtle material incompatibility issues related to the lens coupling fluids used in the lens multiplets (Buckley et al. 2008, Nordsieck et al. 2010). The repaired optics were re-integrated into the spectrograph in July 2009 and the instrument is ready to be lifted to the telescope pending other commissioning tasks.

At the time of writing SALT is in a period of telescope and instrument re-commissioning, due to last into early 2011 with science observations to resume mid-2011. Despite the technical problems described, SALT was used for science observations during the early commissioning and verification phases (late 2005 to early 2009), which has resulted in 30 refereed publications that include SALT observations.

\section{SALTICAM the high-speed camera}

SALTICAM (O'Donoghue et al. 2006) was built at SAAO and was conceived as a multipurpose device used both as an acquisition camera for RSS and a scientific imager. Its optics were designed to allow observations down to the UV atmospheric cut off $(\sim 320 \mathrm{~nm})$. A suite of broad and narrow band filters cover the wavelength range down to the UV. SALTICAM employs frame transfer CCDs, which allows for fast acquisition and imaging. For frame transfer mode, with $2 \mathrm{x} 2$ binning, the shortest exposures are $\sim 2.5 \mathrm{sec}$. A special 
high-speed photometry mode, known as "slot mode", allows for a 20 arcsec wide region of the CCD to be illuminated by moving an occulting mask with a narrow slot. This illuminated region of the CCD is then quickly (typically $\sim 6 \mathrm{~ms}$ ) row-shifted into the storage array, while another exposure begins. Thus, photometry can be achieved of targets (and local comparison stars) with down to $\sim 80 \mathrm{~ms}$ time resolution, with negligible deadtime. Many commissioning programs have already used the high time-resolution mode of SALTICAM very successfully (e.g. O'Donoghue et al. 2006, Woudt et al. 2006, Coe et al. 2007, Günther et al. 2010) while the "normal" full-field imaging has also resulted in succesful studies, image quality problems notwithstanding (e.g. Finkelman et al. 2008, 2010, Kwiatkowski et al. 2009, 2010).

\section{The RSS spectrograph}

The main science workhorse instrument on SALT is the Robert Stobie Spectrograph constructed by the University of Wisconsin-Madison, one of the SALT partners. The RSS is especially designed to: i) observe efficiently down to the atmospheric cut-off; ii) use Volume Phase Holographic transmission gratings with a fully articulating grating and camera to maximize freedom in choosing wavelength ranges in between 320 and $900 \mathrm{~nm}$ and resolutions between $\mathrm{R} 300$ to $\mathrm{R}$ 10000; iii) be a multi-mode instrument. The observing modes include, in addition to long-slit spectroscopy, a multiobject mode (MOS), which allows up to a hundred slitlets to be positioned anywhere within the fieldof-view; a Fabry-Perot imaging spectroscopy; all Stokes mode spectropolarimetry and imaging polarimetry; narrow band imaging; and the use of fast frame-transfer CCDs to allow high-speed (to $10 \mathrm{~Hz}$ ) observations in all modes. Many of the modes can also be combined to use such exotic modes as Fabry-Perot spectropolarimetry. The sensitivity in the extreme blue is one of the niche areas of SALT, a feature rare with large telescopes.

During the brief initial commissioning period of RSS in 2005/2006 before it was taken off the telescope for the throughput repair, RSS was used in a number projects, mostly in long-slit spectroscopic studies of extra-galactic targets (e.g. Brosch et al. 2007, Väisänen et al. 2008, Kniazev et al. 2008,2009). Results from these were encouraging considering the throughput shortfall of the spectrograph and the image quality of the whole telescope at the time and much mire is expected with the repaired system.

An extension for the RSS, a near-IR arm of the instrument, is already in construction at the University of Wisconsin-Madison. The wavelength coverage will be extended through the $J$ and $H$ bands to $\sim 1.7 \mu \mathrm{m}$. Moreover, the optical and NIR beams can be used simultaneously with a dichroic, making RSS at the time of the completion of the NIR arm in 2013, one of the very few instruments anywhere capable of simultaneous spectroscopy over such a wide wavelength range. It is also noteworthy that the same MOS, Fabry-Perot and polarimetric capabilities of RSS will also be available for the NIR arm.

\section{SALT strengths for extragalactic work}

Because of service mode scheduling and limited sky visibility at a given time (an inseason target would typically be visible twice per night for 1-3 hours each time) SALT is ideal for extragalactic surveys where targets are distributed over large areas of sky. While large-area deep extragalactic surveys are not ideal because of the modest $<10$ ' fov and seeing limited (typically $\sim 1$ arcsec at best) imaging, spectroscopic follow-up surveys of a wide range of targets, whether distant galaxy clusters, galaxies, LBGs, QSOs, or $\mathrm{SNe}$, is where SALT is expected to deliver its most significant results. All types of faint targets benefit from the large collecting area, but SALT will be especially competetive for 
diffuse low-surface brightness (LSB) objects where a sub-arcsec point-spread-functions would not be essential. In spectroscopy of faint outer regions of galaxies, for example, or other LSB objects, and especially in the blue part of the spectrum, the SALT/RSS combination is expected to be the best performing instrument anywhere currently.

Studies of e.g. stellar populations and Planetary Nebulae in other galaxies are expected to be commonplace, as well as their kinematics and dynamics. The MOS capability enhances the efficiency of such studies. The blue sensitivity, especially the high-throughput U-band, is very important for both SED (spectral energy distribution) modelling of galaxies, and modelling of ages, metallicities, and extinctions of (super) star clusters in low-redshift galaxies. Finally, the high time-resolution combined with the large collecting area enables (spectral) variability studies of AGN, or even exploring new areas such as spectro-polarimetric variability of SNe and GRBs.

\section{Summary}

SALT is a new major international telescope opening up significant opportunities for African scientists at the cutting edge of modern astrophysical research. African astronomers are strongly encouraged to contact their South African colleagues and SALT to make use of this facility for projects in a wide range of fields. SALT is a service mode telescope, so no travelling is required for observations. Data are delivered within $24 \mathrm{~h}$ of observations, fully or pre-reduced, to make the scientific utilization of them time-efficient.

\section{Acknowledgements}

$\mathrm{PV}$ thanks the conference organizers for an exciting and fruitful time in Ouagadougou. The authors would like to acknowledge the many people who have worked over the years to see SALT and its instruments become a reality, at SALT/SAAO as well as at the various partner institutions.

\section{References}

Brosch, N., et al., 2007, MNRAS, 382, 1809

Buckley, D. A. H.., Swart, G. P., \& Meiring, J. G., 2006, Proc. SPIE 6267, 62670Z

Buckley, et al., 2008, Proc. SPIE 7014, 701407-1

Burgh, E., et al., 2003, Proc. SPIE 4841, 1463

Coe, M. J., et al., 2007, MNRAS, 378, 1427

Crawford, S. M., et al., 2010, Proc. SPIE 7737, 54

Finkelman, I., et al., 2010, MNRAS, 409, 727

Finkelman, I., et al., 2008, MNRAS, 390, 969

Günther, H. M., et al., 2010, A\&SA, 518, 54

Kniazev, A., et al., 2008, MNRAS, 388, 1667

Kniazev, A., et al., 2009, MNRAS, 395, 1121

Kwiatkowski, T., et al., 2009, A\&A, 495, 967

Kwiatkowski, T., et al., 2010, A\& A, 509, 94

Nordsieck, K. H., Nosan, F., \& Shier, J. A., 2010, Proc SPIE 7735, 298

O'Donoghue, D., et al., 2006, MNRAS, 372, 151

O'Donoghue, D., et al., 2008, Proc. SPIE 7018, 13

O'Donoghue, D., et al., 2010, Proc. SPIE 7739, 24

Väisänen, P., et al. 2008, MNRAS, 384, 886

Woudt, P. A., et al., 2006, MNRAS, 371, 1497 